\section{ORLANDO PLAYS HOST TO THE 2017 ASCO GENITOURINARY CANCER SYMPOSIUM}

Over 3 days at the end of February, clinicians and scientists from around the world descended on Florida, to attend the 2017 Genitourinary Cancers Symposium from the American Society of Clinical Oncology (ASCO-GU).

From an attendance of just 900 at its inception, over 3,300 delegates took part in the 2017 meeting. Scientific chairman, William Dahut, opened proceedings by drawing attention to the influence of ASCO-GU on clinical practice: "The interaction between science and patient care is what makes this meeting unique," he commented. "Patients around the world are impacted by this meeting in a very positive way." The meeting made use of an interactive question and answer system, enabling delegates to send their questions directly to the speakers during their presentations, as well as enabling the audience to vote and interact in debates and discussions.

The first session of the congress built on the basis of combining science and patient care by including a patient on the panel for the first time. To start the prostate cancer session, Laurence Klotz compared active surveillance guidelines in the UK, USA, and Canada, and considered the outstanding questions of where to draw the line in Gleason 3+4 disease, the role of imaging and biomarkers, and the role of exercise and lifestyle changes. This presentation was followed by a talk from Freddie Hamdy about the ProtecT study - the largest randomized controlled trial to compare active monitoring, surgery, and radiation therapy for PSA-detected localized prostate cancer. Other highlights of the prostate cancer sessions included Rosalind Eeles' highlighted abstract presentation, which described the identification of 65 novel prostate cancer susceptibility loci from a meta-analysis of $>145,000$ men, and Eliezer Van Allen on the genomic evolution of prostate cancer, which highlighted the soon-to-be-launched patient-led prostate cancer project (www.pcproject.org).

Day 2 of the symposium began with bladder cancer, and started with a talk from Elizabeth Plimack on DNA repair genes. This session also included a presentation on immunonutrition and radical cystectomy from Eugene Lee. In the afternoon, Emma Hall from The Institute of Cancer Research presented data from the BC2001 trial, which investigated synchronous radiation therapy for bladder cancer with a median 10-year follow-up duration. The data showed improved bladder-cancer-specific survival, although the overall survival result did not reach significance.

Also on day 2, a number of talks focussed on the current knowledge of penile cancer. Although rare, penile cancer can have a substantial effect on quality of life, partly owing to the effects of surgical treatment. With this in mind, two talks considered nonsurgical therapy. Vitaly Margulis began by discussing the genomics of penile carcinomas and considering the potential for immunotherapy. A subsequent talk by Jonathan Tward asked why penile cancer is treated differently from other squamous carcinomas - in which surgery is considered a salvage option and primary therapy is nonsurgical by offering surgery as the first-line treatment.

Highlights of the final day of the meeting included a discussion of advanced germ cell tumour (GCT) genetics and the potential role of immunotherapy in patients with this disease, with The Cancer Genome Atlas data on GCT expected later this year. W. Marston Linehan delivered a rousing keynote speech on genetic conditions with a predisposition to renal cell cancer. His assertion that kidney cancer is not one disease, but many - with different histology, course, and response to therapy - that just happen to arise in the same organ, was one of the most tweeted quotes of the meeting.

Presentations from the meeting are available to watch on the ASCO-GU 2017 website (http://gucasym.org). The next ASCO-GU symposium will take place in San Francisco, February 8-10 2018. 